\title{
COVID-19 pandemic in the neonatal intensive care unit: any effect on late-onset sepsis and necrotizing enterocolitis?
}

\author{
Flavia Indrio ${ }^{1}$ - Alessia Salatto ${ }^{1}$. Orsola Amato ${ }^{2} \cdot$ Fabio Bartoli $^{1}$. Letizia Capasso ${ }^{3}$. Luigi Corvaglia ${ }^{4}$. \\ Gianfranco Maffei ${ }^{5} \cdot$ Fabio Mosca $^{2} \cdot$ Massimo Pettoello Mantovani $^{1} \cdot$ Francesco Raimondi $^{3} \cdot$ Matteo Rinaldi $^{5}$. \\ Paola Roggero ${ }^{2} \cdot$ Arianna Aceti $^{4}$ (i)
}

Received: 20 May 2021 / Revised: 27 August 2021 / Accepted: 31 August 2021 / Published online: 6 September 2021

(C) The Author(s), under exclusive licence to Springer-Verlag GmbH Germany, part of Springer Nature 2021

\begin{abstract}
The study was aimed at describing potential indirect effects of pandemic-related measures on very-low-birthweight infants in four Italian NICUs. No overall change in late-onset sepsis (LOS) and necrotizing enterocolitis was documented. However, in the NICU where baseline LOS rate was high, a significant reduction in LOS incidence was recorded.

Conclusion: COVID-19-related implementation of NICU hygiene policies is likely to reduce the occurrence of LOS in high-risk settings.
\end{abstract}

\section{What is Known:}

- COVID-19 pandemic has disrupted routine care in Neonatal Intensive Care Units (NICUs), mostly by tightening infection control measures and restricting parental presence in the NICU.

- Beyond the described psychological impact of COVID-19 related measures on healthcare workers and NICU families, their consequences in terms of preterm infants' clinical outcomes have not been described in detail yet.

What is New:

- Strengthened infection-control measures do not seem to have an overall influence on the incidence of necrotising enterocolitis and late-onset sepsis in very-low-birth-weight infants.

- However, the implementation of these measures appears to reduce the occurrence of late-onset sepsis in settings where the baseline incidence of the disease is high.

Communicated by Daniele De Luca.

Arianna Aceti

arianna.aceti2@unibo.it

Flavia Indrio

flaviaindrio1@gmail.com

Alessia Salatto

alessiasalatto1@gmail.com

Orsola Amato

orsola.amato@policlinico.mi.it

Fabio Bartoli

fabio.bartoli@unifg.it

Letizia Capasso

letizia.capasso@gmail.com

Luigi Corvaglia

luigi.corvaglia@unibo.it

Gianfranco Maffei

gfmaffei@tiscali.it

Fabio Mosca

fabio.mosca@unimi.it

Massimo Pettoello Mantovani massimo.pettoellomantovani@unifg.it
Francesco Raimondi

raimondi@unina.it

Matteo Rinaldi

matrinaldi@gmail.com

Paola Roggero

paola.roggero@unimi.it

1 Department of Medical and Surgical Sciences, University of Foggia, Foggia, Italy

2 Neonatal Intensive Care Unit, Department of Clinical Science and Community Health, Fondazione I.R.C.C.S. Ca Granda Ospedale Maggiore Policlinico, University of Milan, Milan, Italy

3 Division of Neonatology, Section of Pediatrics, Department of Translational Medical Sciences, "Federico II" University, Naples, Italy

4 Department of Medical and Surgical Sciences - Neonatal Intensive Care Unit, University of Bologna - IRCCS Azienda Ospedaliero-Universitaria di Bologna, Via Massarenti, 11, 40138 Bologna, Italy

5 Neonatal Intensive Care Unit, Ospedali Riuniti, Foggia, Italy 
Keywords COVID-19 - Neonatal intensive care unit . Infection control · Preterm infant · Late-onset sepsis . Necrotizing enterocolitis

$\begin{array}{ll}\text { Abbreviations } \\ \text { GA } & \text { Gestational age } \\ \text { LOS } & \text { Late-onset sepsis } \\ \text { NEC } & \text { Necrotizing enterocolitis } \\ \text { NICU } & \text { Neonatal intensive care unit } \\ \text { SD } & \text { Standard deviation } \\ \text { VLBW } & \text { Very low birth weight } \\ \text { VON } & \text { Vermont Oxford Network }\end{array}$

\section{Introduction}

Since December 2019, the novel coronavirus species SARS$\mathrm{CoV}-2$ has been described and recognized as responsible for the global pandemic which is still affecting human health and healthcare systems worldwide [1].

The clinical presentation of the disease known as COVID-19 is described to be milder in children than in adults; within the paediatric population, younger children appear to be more vulnerable to the infection, especially if having concomitant risk factors [2]. As for the impact of COVID-19 on neonatal care, great effort has been made to protect the mother-infant dyad whenever possible, by preserving rooming-in and breastfeeding practices [3]. However, the pandemic has disrupted usual care processes in Neonatal Intensive Care Units (NICUs), mostly by tightening infection-control measures and restricting parental presence in the NICU, in the attempt to further protect preterm and critically ill newborns [4].

So far, indirect effects of the pandemic in the NICU have been described: these include mainly the psychological distress that separation induces in parents who have already experienced the traumatic effect of their infant's NICU admission, and the obstacles in putting family-centred care into practice [5].

Recently, it has been reported, in a single-centre study performed in a Canadian NICU during the pandemic period, a sudden and unexpected increase in the rate of central-line associated bloodstream infections compared to the pre-pandemic period; this was attributed to the shortages of alcohol-based hand rubs due to a concomitant reduction of their availability at the hospital level. The re-implementation of infection-control measures brought the infection rate back to the pre-pandemic baseline [4]. Furthermore, in a very recent paper, a reduction in the colonization with multiresistant organisms in infants admitted to an Italian NICU was described, following the reinforcement of infection-control measures after COVID19 breakout [6].

To date, there is no other specific evidence of the actual impact of pandemic-related measures on the clinical outcomes of preterm infants admitted to the NICU. In the present study, we aimed to describe the potential effect of the pandemic-related measures on the incidence of late-onset sepsis (LOS) and necrotizing enterocolitis (NEC) in infants with very-low-birthweight (VLBW) and/or born between 22 and 29 weeks gestational age (GA) admitted to four Italian NICUs during the COVID-19 period.

\section{Materials and methods}

A retrospective, observational, multicentre study, involving four Italian NICUs located in different geographic areas of the country (Milan, Bologna, Naples, and Foggia), was performed. All the study NICUs routinely take care of very preterm and VLBW infants. The number of infants born in each study hospital per year ranges from approximately 2500 (NICU C) to 5500 (NICU A). Infants with VLBW and/or GA between 22 and 29 weeks admitted to the study NICUs each year range from approximately 40 (NICU D) to 140 (NICU A). All the NICUs have access to a wide range of paediatric specialists [level III B or III C NICU according to the Vermont Oxford Network (VON) registry definition]. The infant/nurse ratio ranges from $2 / 1$ to $3 / 1$ for the proper NICU cots, and from $6 / 1$ to $4 / 1$ for babies in the high-dependency unit.

Demographic and clinical data of preterm infants born in 2019 and 2020 with VLBW and/or GA between 22 and 29 weeks, which are routinely collected and included anonymously in the VON registry, were evaluated to compare the incidence of LOS and NEC, defined as in the 2020 VON Manual of Operations (Part 2, Release 24.0), between a pandemic (year 2020) and a pre-pandemic period (year 2019). Data obtained from the VON registry were checked against each hospital electronic records, from which additional clinical data (i.e. LOS aetiology) were collected.

The study was conducted in conformity with the principles and regulations of the Helsinki Declaration. Data collection within the VON registry was already part of routine care of each study NICU. The local review board approved the study protocol.

Statistical analyses were performed using IBM SPSS Statistic v.20. The chi-square test was used to compare the incidence of LOS and NEC between periods, both in the overall population and in each study centre. A $P$ value $<0.05$ was considered statistically significant. 
Table 1 Demographic characteristics of preterm infants with gestational age $\leq 29$ weeks and/or very-low birth weight who experienced late-onset sepsis and necrotizing enterocolitis during the study period in four Italian neonatal intensive care units

\begin{tabular}{llllll}
\hline & Overall & Centre A & Centre B & Centre C & Centre D \\
\hline $\begin{array}{l}\text { Number of infants } \\
\text { Late-onset sepsis }\end{array}$ & 572 & 244 & 92 & 157 & 79 \\
$\begin{array}{l}\text { Number of episodes } \\
\text { Gestational age, weeks }\end{array}$ & 115 & 39 & 13 & 55 & 8 \\
Birth weight, grams & $937(276)$ & $895(270)$ & $802(327)$ & $1006(266)$ & $889(205)$ \\
Male/female & $71 / 44$ & $26 / 13$ & $7 / 6$ & $34 / 21$ & $4 / 4$ \\
Necrotizing enterocolitis & & & & & \\
Number of episodes & 26 & 6 & 8 & 1 & 11 \\
Gestational age, weeks & $28(3)$ & $29(4)$ & $28(2)$ & 29 & $28(3)$ \\
Birth weight, grams & $1077(323)$ & $1217(418)$ & $1003(305)$ & 1200 & $1044(300)$ \\
Male/female & $12 / 14$ & $3 / 3$ & $5 / 3$ & $0 / 1$ & $4 / 7$ \\
\hline
\end{tabular}

Values are reported as mean (standard deviation) or numbers as appropriate

\section{Results}

Data from 572 infants with GA $\leq 29$ weeks and/or VLBW were reviewed; recruited infants had a mean GA of 28 weeks (SD 3 weeks) and a mean birth weight (BW) of $1077 \mathrm{~g}$ (SD $343 \mathrm{~g}$ ). None of the mothers had tested positive for COVID19 infection during pregnancy. Demographic characteristics of the studied infants are shown in Table 1. No difference in GA or BW was documented among centres.

Overall, 115 infants experienced LOS, and 26 experienced NEC during the whole study period. Data on LOS and NEC incidence across study centres and study periods are reported in Table 2. No significant difference in LOS or NEC incidence was documented in the overall population. However, in the pandemic period, a significant reduction in LOS incidence was documented in one centre (centre C, $26.6 \%$ vs. $43.6 \%$ in the pre-pandemic period, $P<0.05$ ), and in NEC incidence in another (centre A, no NEC cases vs. $5.4 \%$ in the pre-pandemic period, $P<0.01)$.

As for LOS aetiology, most cases $(62 / 115,54 \%)$ were attributable to coagulase-negative staphylococci, followed by Klebsiella $(15 / 115,13 \%)$, Enterobacter and Staphylococcus aureus (10/115, 8.7\% each). The proportion of LOS episodes attributable to each pathogen was not significantly different in the two periods.

\section{Discussion}

According to the results of the present study, the pandemicrelated measures did not affect the overall incidence of LOS and NEC. However, in the NICU where the baseline LOS rate was high, a significant reduction in the disease incidence was documented.

To evaluate in deeper detail the reasons behind this observation, data about LOS in the three previous years were reviewed. During 2019, the incidence of LOS in NICU C was higher than the previous years. To some extent, this was probably attributable to a transient reduction in the number of NICU nurses, with a consequent increase in the infant/ nurse ratio. This issue was partially solved in 2020, and this could have contributed to the lower incidence of LOS during that year in NICU C. Even if we cannot detail the specific contribution of nurses' number and COVID-related measures to LOS reduction, these data reinforce the observation according to which a strict infection-control bundle, which comprises both personnel implementation and specific infection-control measures, can contribute to LOS reduction among VLBW infants in settings with a high incidence of the disease.

On the contrary, the reason for the reduction in the NEC rate observed in NICU A is unclear, as no significant changes
Table 2 Number of infants, overall and for each study centre, who experienced an episode of late-onset sepsis (LOS) or necrotizing enterocolitis (NEC) in the two study periods (2019, prepandemic and 2020, pandemic)

\begin{tabular}{llllllll}
\hline & LOS & \multicolumn{6}{l}{ NEC } \\
\cline { 2 - 3 } & 2019 & 2020 & $P$ value & & 2019 & 2020 & $P$ value \\
\hline Overall & $63 / 281(22.4 \%)$ & $52 / 291(17.9 \%)$ & 0.1771 & & $13 / 281(4.6 \%)$ & $13 / 291(4.5 \%)$ & 1.000 \\
Centre A & $18 / 112(16.1 \%)$ & $21 / 132(15.9 \%)$ & 1.000 & & $6 / 112(5.4 \%)$ & $0 / 132(0 \%)$ & $\mathbf{0 . 0 0 8 7}$ \\
Centre B & $8 / 51(15.7 \%)$ & $5 / 41(12.2 \%)$ & 0.7669 & & $4 / 51(7.8 \%)$ & $4 / 41(9.8 \%)$ & 1.000 \\
Centre C & $34 / 78(43.6 \%)$ & $21 / 79(26.6 \%)$ & $\mathbf{0 . 0 3 0 2}$ & & $0 / 78(0 \%)$ & $1 / 79(1.3 \%)$ & 1.000 \\
Centre D & $3 / 40(7.5 \%)$ & $5 / 39(12.8 \%)$ & 0.4814 & & $3 / 40(7.5 \%)$ & $8 / 39(20.5 \%)$ & 0.1149 \\
\hline
\end{tabular}

Values are reported as number (percentage). Significant $P$ values $(P<0.05)$ are in bold 
in the NICU standard of care, apart from the COVID-related measures, had occurred during the study period. We cannot exclude that those measures could have had an indirect impact on gut microbiota of VLBW infants admitted to the NICU, thus affecting the occurrence of NEC, but we have no specific data to support this hypothesis.

The ongoing pandemic has disrupted neonatal care worldwide, especially for smallest and critically ill infants [7]. Changes in neonatal care include restriction of parental presence and implementation of protective measures aimed at reducing the likelihood of viral spread in the NICU. It is not clear whether and how these measures might influence the occurrence of neonatal infections. It has been shown by surveillance data from the National Institute of Child Health and Human Development Neonatal Network over a 20-year period that $20-25 \%$ of VLBW infants experience an episode of LOS; the incidence of the disease has decreased over time, with rates as low as $10-15 \%$ documented recently in most neonatal VLBW registries [8].

The cornerstones for reducing LOS rates are strict adherence to established infection-control protocols and minimization of invasive procedures in the NICU [9]. Putting in place effective sepsis reduction bundles might have effects which go well beyond the mere reduction of LOS incidence, including also improved neurodevelopment [10].

To our knowledge, the present study is the first to provide specific data about the potential impact of pandemic-related, infection-control measures on preterm infants' clinical outcomes in the NICU; however, the relatively small number of infants and the limited observation time must be acknowledged, and caution should be used in generalizing these data.

\section{Conclusion}

Despite the acknowledged study limitations, we can hypothesise that one or more COVID-related hygiene measures might have acted in adjunct to environmental and contact precautions which were already in place, thus potentially contributing at reducing LOS rate in the NICU where the baseline incidence of the disease was high. On the contrary, these measures had no significant effect in settings where LOS rate was already quite low and did not seem to affect substantially the incidence of NEC. It is not clear which specific intervention(s) could have contributed most to sepsis reduction, and future studies should hopefully explore this issue.

Authors' contributions FI, LC, FM, FR, and PR conceived the study; AS, OA, FB, LC, MPM, MR, GM, and AA collected the data. FI and AA analysed and interpreted the data; they also wrote the first draft of the paper, which was revised critically by all the other authors. All the authors gave final approval of the version to be submitted.

Funding The authors did not receive support from any organization for the submitted work.

Data availability Data about included infants are routinely recorded by all the study centres within the Vermont Oxford Network (VON) registry.

Code availability N/A

\section{Declarations}

Conflict of interest The authors have no relevant financial or non-financial interests to disclose.

Ethical approval The study was conducted in conformity with the principles and regulations of the Helsinki Declaration. Data collection within the Vermont Oxford Network (VON) registry was already part of routine care of each study NICU. The local review board approved the study protocol.

Consent to participate and to publish Study data were collected from the VON Registry, which was already part of routine care of each study NICU.

\section{References}

1. World Health Organization (2021) WHO COVID dashboard. https://covid19.who.int/. Accessed 8 Apr 2021

2. Dong Y, Mo X, Hu Y, Qi X, Jiang F, Jiang Z, Tong S (2020) Epidemiology of COVID-19 among children in China. Pediatrics 145:e20200702. https://doi.org/10.1542/peds.2020-0702

3. Ronchi A, Pietrasanta C, Zavattoni M et al (2021) Evaluation of rooming-in practice for neonates born to mothers with severe acute respiratory syndrome coronavirus 2 infection in Italy. JAMA Pediatr 175:260-266. https://doi.org/10.1001/jamapediatrics. 2020.5086

4. Kharrat A, Neish A, Diambomba Y, Jain A (2021) Non-COVID co-morbidity: potential indirect consequences of the SARS-CoV-2 pandemic in a neonatal intensive care unit. J Hosp Infect 109:6567. https://doi.org/10.1016/j.jhin.2020.12.010

5. Cena L, Biban P, Janos J, Lavelli M, Langfus J, Tsai A, Youngstrom EA, Stefana A (2021) The collateral impact of COVID-19 emergency on neonatal intensive care units and family-centered care: challenges and opportunities. Front Psychol 12:630594. https://doi. org/10.3389/fpsyg.2021.630594

6. Pezzotta R, De Francesco MA, Caruso A (2021) Possible impact of severe acute respiratory syndrome coronavirus- 2 control measures in reducing colonization by gram-negative bacteria and Candida spp. in a neonatal intensive care unit. Pediatr Infect Dis J 40:e211-e213. https://doi.org/10.1097/INF.0000000000003092

7. Rao SPN, Minckas N, Medvedev MM et al (2021) Small and sick newborn care during the COVID-19 pandemic: global survey and thematic analysis of healthcare providers' voices and experiences. BMJ Glob Health 6:1-11. https://doi.org/10.1136/ bmjgh-2020-004347

8. Ramasethu J (2017) Prevention and treatment of neonatal nosocomial infections. Matern Health Neonatol Perinatol 3:1-11. https:// doi.org/10.1186/s40748-017-0043-3 
9. Dong Y, Speer CP (2015) Late-onset neonatal sepsis: recent developments. Arch Dis Child Fetal Neonatal Ed 100:F257-F263. https://doi.org/10.1136/archdischild-2014-306213

10. Davis JW, Odd D, Jary S, Luyt K (2016) The impact of a sepsis quality improvement project on neurodisability rates in very low birthweight infants. Arch Dis Child Fetal Neonatal Ed 101:F562F564. https://doi.org/10.1136/archdischild-2015-309804

Publisher's Note Springer Nature remains neutral with regard to jurisdictional claims in published maps and institutional affiliations. 